\title{
Coupled time discretisation of dynamic damage models at small strains 12
}

\author{
Tomáš Roubíček 3
}

\begin{abstract}
The dynamic damage model in viscoelastic materials in Kelvin-Voigt rheology is discretised by a scheme which is coupled, suppresses spurious numerical attenuation during vibrations, and has a variational structure with a convex potential for small time-steps. In addition, this discretisation is numerically stable and convergent for the time step going to zero. When combined with the FEM spatial discretisation, it leads to an implementable scheme and to that iterative solvers (e.g. the Newton-Raphson) used for the nonlinear algebraic systems at each time level have guaranteed global convergence. Models which are computationally used in some engineering simulations in a non-reliable way are thus stabilized and theoretically justified in this viscoelastic rheology. In particular, this model and algorithm can be used in a reliable way for a dynamic fracture in the usual phase-field approximation.
\end{abstract}

Keywords: evolution variational inequalities, damage, fracture, phase-field approximation, numerical approximation, implicit monolithic time discretisation.

AMS Classification: 35R45, 65K15, 74H15, 74R05, 74R10.

\section{Introduction}

Damage (possibly interpreted as a phase-field approximation of fracture) is an important phenomenon in continuum mechanics of solids and many models have been devised in engineering and partly also analyzed in mathematical literature, cf. e.g. [24,42] and references therein. Mostly, a scalar-valued damage field is considered. Historically, this concept dates back to Kachanov [20], and has been widely used both at finite and at small strains, cf. e.g. the monographs [16, 21, 29, 46, 47].

In agreement with experiments, one other aspect is often built into damage models, namely an internal length scale through the gradient of damage, cf. [3, 16], and, in some situations, also inertial effects. The former aspect expresses certain nonlocality in the sense that damage of a particular spot is to some extent influenced by its surrounding, leading to possible hardening or softening-like effects and, by introducing a certain internal length scale, eventually prevents damage microstructure development. The latter aspect is often important, in particular because damage may evolve very fast and elastic waves or vibrations

\footnotetext{
${ }^{1}$ This is an author accepted version published on line on April 2019 in IMA Journal of Numerical Analysis, DOI 10.1093/imanum/drz014, later as (2020) 40, 1772--1791, and placed on arXiv in accord with the "Standard Licence"' of the Oxford University Press.

${ }^{2}$ URL https://academic.oup.com/imajna/article-abstract/40/3/1772/5431182,

3 Institute of Thermomechanics of the Czech Acad. Sci., Dolejškova 5, CZ-182 08 Praha 8, Czech Republic, email: tomas.roubicek@mff.cuni.cz
} 
may vitally accompany the damage process itself, and also the finite speed of propagation of information naturally avoids nonphysical long-range interactions sometimes involved in quasistatic damage models. This happens in geophysics during ruptures of lithospheric faults when seismic waves are emitted or in material testing in engineering. In some other situations, the external load may be extremely fast (e.g. during explosions or impacts of projectiles or asteroids) so that dynamical effects may play a vital role.

Although the split (called also fractional-step, or staggered) schemes are robust and even may conserve energy, cf. also [30, 33, 41, 43] or [32, Sect.5.1-5.2], the fully-coupled scheme are often used in engineering inspite of no guaranty for numerical stability and convergence, or even for a global convergence of iterative solvers which must be necessarily used for (generally) nonconvex incremental problems arising at each time level. To the last point, typically the Newton-Raphson or the alternate minimization algorithm (AMA) are used. Especially the former option is explicitly reported in literature as unreliable in the context of damage or phase-field crack models, cf. [6, 13, 35, 44, 48, 49], although one can even find an opposite (not correct in general) belief and a (rather particular) experience that a fully coupled monolithic solution can even prevent numerical instabilities or converges, cf. e.g. 37, 38. As a matter of fact, the Newton-Raphson algorithm, if converges, can find only a general critical points which does not need to be physically relevant and cannot yield apriori estimates and thus numerical stability of the discretisation scheme.

Let us only mention that the AMA procedure works differently because it monotonically increases energy and, if converges, it founds critical points which minimize the energy at least separately at the displacement and at the damage variables, cf. [7] for a theoretical analysis. The relation of AMA and the Newton-Raphson algorithm is discussed in [10]. Sometimes, the variant of AMA which updates the damage constraints within each iteration is used, which is then basically the staggered scheme, cf. [22]. Cf. also [14] for a comparison and some other variants.

Sometimes, attempt for stabilization of such unreliable schemes has intuitively been done by inventing some viscosity into the damage flow rule [23, 28, 31, 52], although even this is not reliable, cf. Remark 3.3. For some others, even more phenomenological and theoretically unjustified attempts to cope with failing iterative solvers, see e.g. [35].

The goal of this article is to investigate a true physically-motivated stabilization by means of the viscosity in the bulk, i.e. by inventing Kelvin-Voigt rheology instead of a purely elastic model, or a less robust stabilization by mere inertia in a purely elastic model, cf. Remark 5.3. This viscosity has a physical motivation in particular during fast crack growth or fast loading, cf. the discussion in [17, \$8.2]. The fully-coupled schemes thus become amenable for a-priori estimates (numerical stability) and their convergence can be shown, too. In addition, we keep the variational structure of the incremental problems and, for small time step, the guaranty of global convergence of iterative numerical algorithms.

It should be emphasized that fully coupled (also, in engineering literature, sometimes called monolithic) schemes do not rely on component-wise convex (or even quadratic) structure and, if working, they can be used for more general problems without any algorithmic changes, in contrast to split schemes that typically take a benefit from implementation of quadratic-programming. In particular, it concerns the very natural phenomenon (not considered here) that damage may act very differently on compression than on tension so that the stored energy is not quadratic in terms of strains. Even stored energies nonconvex in terms 
of strains are considered in some applications in rupturing-rock mechanics, cf. [19, 27] and references therein. This is a certain advantage of the monolithic schemes, beside their more straightforward implementation in some software packages as e.g. Abaqus, cf. e.g. [26, 34].

The plan of this paper is following: The model is formulated in Sect. 2 by specifying the governing energies and by using the Hamilton variational principle. Its time discretisation is devised in Sect. 3 and its basic properties are proved, namely the variational structure, existence of discrete solutions, and convexity of the governing potentials for sufficiently small time steps. In Sect. 4, the numerical stability analysis (i.e. a-priori estimates) of the timediscrete model is performed, and its convergence towards the original continuous model is proved. Eventually, the paper ends with several remarks in Sect. 5 outlining the phase-field fracture, and various modifications and further usage.

\section{The model of damage at small strains}

We deal with a relatively simple model of damage in Kelvin-Voigt viscoelastic solid without influencing the viscous part. We consider also inertial effects, so that we can capture also vibrations or waves that can be generated during fast damage or rupture. Yet, our results do not rely on the presence of inertia so that the quasistatic variant is covered, too.

The independent variables of the model are the displacement $u: \Omega \rightarrow \mathbb{R}^{d}$ and the damage $\alpha: \Omega \rightarrow[0,1]$, both varying in time, with $\Omega \subset \mathbb{R}^{d}$ being a fixed bounded domain in $\mathbb{R}^{d}$ with a Lipschitz boundary $\Gamma, d=2,3$. The viscoelastic material is considered with the viscous response undergoing (quite naturally) damage in the same way as the elastic response. The mass density $\varrho$ which is responsible for inertia is naturally independent of damage because the mass is not destroyed by damaging of interatomic links.

The rational-mechanical approach builds models on the base of energies. Here, we want to use a model governed by the energies:

$$
\begin{array}{lll}
\text { stored energy: } & \mathscr{E}(u, \alpha):=\int_{\Omega} \frac{1}{2} \mathbb{C}(\alpha) e(u): e(u)-\phi(\alpha)+\frac{\kappa}{p}|\nabla \alpha|^{p} \mathrm{~d} x, \\
\text { dissipation potential: } & \mathscr{D}(\alpha ; v, \dot{\alpha}):=\int_{\Omega} \frac{1}{2} \mathbb{D}(\alpha) e(v): e(v)+\zeta(\dot{\alpha}) \mathrm{d} x, \\
\text { kinetic energy: } & \mathscr{T}(v):=\int_{\Omega} \frac{\varrho}{2}|v|^{2} \mathrm{~d} x, \\
\text { external loading: } & \mathscr{F}(t, u):=\int_{\Omega} f(t) \cdot u \mathrm{~d} x+\int_{\Gamma} g(t) \cdot u \mathrm{~d} S,
\end{array}
$$

where $\varrho>0$ is the mentioned mass density, the variable $v$ is the placeholder for velocity $\dot{u}$, i.e. the time derivative of $u, e(u)=\frac{1}{2} \nabla u^{\top}+\frac{1}{2} \nabla u$ the small-strain tensor, $\phi=\phi(\alpha)$ is the stored energy of damage reflecting the phenomenon that, on a microscopical level, damage means some microcracks or microvoids which increase micro-surfaces inside material and thus its internal stored energy. Thus, physically, $\phi$ is increasing or at least nondecreasing. The negative sign in (1a) is thus related with the convention that $\alpha=1$ means no damage while $\alpha=0$ means maximal damage.

Based on the energies (1), the evolution is governed by the Hamilton variational principle generalized for nonconservative systems. More specifically, defining the Lagrangian 
$\mathscr{L}(v, u, \alpha)$ and the nonconservative dissipative force $\mathscr{F}_{\text {diss }}(v, \alpha, \dot{\alpha}):=\mathscr{D}_{(v, \dot{\alpha})}^{\prime}(\alpha ; v, \dot{\alpha})$ by

$$
\begin{aligned}
& \mathscr{L}(t, v, u, \alpha)=\mathscr{T}(v)-\mathscr{E}(u, \alpha)-\mathscr{F}(t, u), \\
& \left\langle\mathscr{F}_{\text {diss }}(v, \tilde{\alpha}, \dot{\alpha}),(u, \alpha)\right\rangle=\int_{\Omega} \mathbb{D}(\tilde{\alpha}) e(v): e(u)+\zeta^{\prime}(\dot{\tilde{\alpha}}) \alpha \mathrm{d} x .
\end{aligned}
$$

The evolution on a fixed time interval $I=[0, T]$ will be a trajectory $t \mapsto(u(t), \alpha(t))$ with $t \in I$ forming a critical point of the functional

$$
(u, \alpha) \mapsto \int_{0}^{T} \mathscr{L}(t, \dot{u}, u, \alpha)-\left\langle\mathscr{F}_{\text {diss }}(v, \tilde{\alpha}, \dot{\tilde{\alpha}}),(u, \alpha)\right\rangle \mathrm{d} t
$$

provided $v=\dot{u}$ and $\tilde{\alpha}=\alpha$. For $\mathscr{F}_{\text {diss }}=0$, this is known as the Hamilton variational principle for conservative systems. The extension (3) for nonconservative dissipative force $\mathscr{F}_{\text {diss }} \neq 0$ is a bit formal, cf. [4].

From (3), we obtain the system of partial differential equations (or inclusions):

$$
\begin{array}{ll}
\varrho \ddot{u}-\operatorname{div}(\mathbb{C}(\alpha) e(u)+\mathbb{D}(\alpha) e(\dot{u}))=f & \text { in } Q, \\
\partial \zeta(\dot{\alpha})+\frac{1}{2} \mathbb{C}^{\prime}(\alpha) e(u): e(u)-\operatorname{div}\left(\kappa|\nabla \alpha|^{p-2} \nabla \alpha\right) \ni \phi^{\prime}(\alpha) & \text { in } Q,
\end{array}
$$

where $Q:=I \times \Omega$, accompanied by the boundary conditions $(\mathbb{C}(\alpha) e(u)+\mathbb{D}(\alpha) e(\dot{u})) \vec{n}=g$ and $\nabla \alpha \cdot \vec{n}=0$ on $\Sigma:=I \times \Gamma$, with $\vec{n}$ denoting the outward unit normal to $\Gamma$. The notation " $\partial \zeta$ " stands for the usual convex subdifferential, i.e. formally (4b) means the inequality

$$
\zeta(z)+\left(\frac{1}{2} \mathbb{C}^{\prime}(\alpha) e(u): e(u)-\operatorname{div}\left(\kappa|\nabla \alpha|^{p-2} \nabla \alpha\right)-\phi^{\prime}(\alpha)\right)(z-\dot{\alpha}) \geq \zeta(\dot{\alpha})
$$

to be valid on $Q$ for all $z \in \mathbb{R}$.

Throughout this article, we will accept the modelling assumptions

$$
\begin{aligned}
& \mathbb{C}^{\prime}(0)=0, \quad \phi^{\prime}(0) \geq 0, \quad \text { and } \quad \zeta(\dot{\alpha})=+\infty \text { for } \dot{\alpha}>0, \\
& \mathbb{D}(\alpha)=\mathbb{D}_{0}+\chi_{\mathrm{R}} \mathbb{C}(\alpha) \quad \text { with } \quad \chi_{\mathrm{R}}>0 \text { a fixed relaxation time. }
\end{aligned}
$$

The assumption (6a) ensure that the evolution of damage $\alpha$ is unidirectional in the sense that healing $\dot{\alpha}>0$ is not allowed, and that $\alpha$ stays valued in $[0,1]$ within the evolution provided it is such at the initial time, so that these constraints $0 \leq \alpha \leq 1$ do not need to be considered explicitly, which simplifies the formulation as well as the analysis. Like in [25], (6b) represents a bit special but not much application-restricting case that viscous dissipative processes are of the same character as the elastic storage. Some results (in fact, all except Proposition 4.4) would hold even without assuming (6b). In fact, (6b) is only needed for proving a strong convergence of the strains without relying on the energy conservation in the damage flow rule, which is not guaranteed in our rate-dependent uni-directional damage model (in contrast to the rate-independent model as in [25] or [32, Sect. 5.2.5]).

The energetics of the system (44) is revealed when testing (4a) by $\dot{u}$ and (4a) by $\dot{\alpha}$. This gives, at least formally (i.e. it the solution would be smooth enough so that $\operatorname{div}\left(|\nabla \alpha|^{p-2} \nabla \alpha\right)$ would be in $L^{2}(Q)$ and thus in duality with $\dot{\alpha}$ ), the energy balance

$$
\mathscr{T}(\dot{u}(t))+\mathscr{E}(u(t), \alpha(t))+\int_{0}^{t} \Xi(\alpha ; \dot{u}, \dot{\alpha}) \mathrm{d} t=\mathscr{T}\left(v_{0}\right)+\mathscr{E}\left(u_{0}, \alpha_{0}\right)+\int_{0}^{t} \mathscr{F}(t, \dot{u}) \mathrm{d} t
$$


where the dissipation rate is

$$
\Xi(\alpha ; v, \dot{\alpha})=v \cdot \partial_{v} \mathscr{D}(\alpha ; v)+\dot{\alpha} \partial_{\dot{\alpha}} \mathscr{D}(\dot{\alpha})=\int_{\Omega} \mathbb{D}(\alpha) e(v): e(v)+\dot{\alpha} \partial \zeta(\dot{\alpha}) \mathrm{d} x .
$$

Typically, $\zeta$ is smooth except only at $\dot{\alpha}=0$, so that $\dot{\alpha} \partial \zeta(\dot{\alpha})$ is well defined as a single-valued function on $Q$.

In terms of the variable $v$ for velocity, instead of the 2 nd-order force-equilibrium equation (4a) involving the inertial force $\varrho \ddot{u}$, we rewrite (44) in the form of a 1st-order system

$$
\begin{array}{ll}
\dot{u}=v & \text { in } Q, \\
\varrho \dot{v}-\operatorname{div}\left(\mathbb{D}_{0} v+\mathbb{C}(\alpha) e\left(u+\chi_{\mathrm{R}} v\right)\right)=f & \text { in } Q, \\
\partial \zeta(\dot{\alpha})+\frac{1}{2} \mathbb{C}^{\prime}(\alpha) e(u): e(u)-\operatorname{div}\left(\kappa|\nabla \alpha|^{p-2} \nabla \alpha\right) \ni \phi^{\prime}(\alpha) & \text { in } Q .
\end{array}
$$

We consider the initial-boundary value problem

$$
\begin{array}{ll}
\left(\mathbb{D}_{0} v+\mathbb{C}(\alpha) e\left(u+\chi_{\mathrm{R}} v\right)\right) \vec{n}=g \quad \text { and } \quad \nabla \alpha \cdot \vec{n}=0 & \text { on } \Sigma, \\
u(0)=u_{0}, \quad \dot{u}(0)=v_{0}, \quad \alpha(0)=\alpha_{0} & \text { on } \Omega .
\end{array}
$$

A very similar model has already been analysed by C.J. Larsen, C. Ortner, and E. Süli by using the semi-implicit (fractional-step) time-discretisation in [25] for the rate-independent unidirectional damage, cf. also [32, Sect.5.1.1 and 5.2.5]. In fact, for a special choice of the damage energy $\phi$, and for $p=2$, our model nearly equals to that one in [25] except that we consider a rate-dependent damage evolution, in contrast to [25] where a rate-independent damage is considered. Yet, to gain the variational structure of our monolithic time disretisation, we will make a semi-implicit discretisation of the viscous term and will need $p>d$ in the proof of convergence in Proposition 4.4. Of course, the choice $p=2$ is more straightforward and common in engineering computations. Here, let us only remark that a compromise model replacing $|\nabla \alpha|^{p-2}$ in (9) by $1+\varepsilon|\nabla \alpha|^{p-2}$ with some (pressumably small) $\varepsilon>0$ and again $p>d$ would work, too.

The rate-independent models represent a certain asymptotical abstraction for slow external loading activating internal processes which may evolve much faster, and can sometimes be simpler than their rate-dependent variants, cf. [32, Example 1.2.7] for a damage problem. Even the rate-dependent model allows for analytical existence result, cf. [24, 42] for $p=2$; the existence results presented here are only new for $p \neq 2$. Yet, our focus is on numerical approximation scheme and on its stability and convergence. In these aspects, there are several differences comparing to [25] beside rate-dependent variant of the damage-flow rule: the fully coupled scheme is used here (while [25] uses the split, staggered scheme) and the inertial term is here discretised in an energy-conservative way to suppress spurious numerical attenuation during vibrations which would otherwise effectively kill computational simulations of such problems.

\section{Coupled implicit time discretisation}

Although for some $\mathbb{C}(\cdot)$ and $\mathbb{D}(\cdot)$ the functionals (1a) and $(1 \mathrm{~b})$ can be convex, the damage models inevitably need to work with nonconvex energies. This nonconvexity is responsible 
for the desired phenomenon of sudden damage/rupture. Some engineering algorithms intentionally want to keep this nonconvexity even on the discrete level, exploiting also that software packages as e.g. Abaqus accent rather coupled, so-called monolithic scheme, cf. e.g. [26].

We apply here the coupled implicit time discretisation to the system (9) rather than (4) in a particular way. Namely:

- we discretise the inertial part by the mid-point (Crank-Nicolson) formula rather than the backward Euler one in order to reduce unwanted numerical attenuation which would otherwise be exhibited by the implicit 2nd-order scheme, and

- we intentionally use the fully implicit backward-Euler formula for the stored-energy terms to be as close as possible to the usual engineering implementation,

- we use a semi-implicit (but not the fully implicit backward-Euler) formula for the viscous stress $\mathbb{D}(\alpha) e(v)$ in order to keep the variational structure of the incremental problems, cf. Proposition 3.2 below.

The resulting iterative coupled boundary-value problems here are:

$$
\begin{gathered}
\frac{u_{\tau}^{k}-u_{\tau}^{k-1}}{\tau}=v_{\tau}^{k-1 / 2} \quad \text { with } \quad v_{\tau}^{k-1 / 2}:=\frac{v_{\tau}^{k}+v_{\tau}^{k-1}}{2}, \\
\varrho \frac{v_{\tau}^{k}-v_{\tau}^{k-1}}{\tau}-\operatorname{div}\left(\mathbb{C}\left(\alpha_{\tau}^{k}\right) e\left(u_{\tau}^{k}\right)+\mathbb{D}\left(\alpha_{\tau}^{k-1}\right) e\left(v_{\tau}^{k-1 / 2}\right)\right)=f_{\tau}^{k} \\
\text { with } \quad f_{\tau}^{k}:=\int_{(k-1) \tau}^{k \tau} f(t) \mathrm{d} t, \quad \text { and } \\
\partial \zeta\left(\frac{\alpha_{\tau}^{k}-\alpha_{\tau}^{k-1}}{\tau}\right)+\frac{1}{2} \mathbb{C}^{\prime}\left(\alpha_{\tau}^{k}\right) e\left(u_{\tau}^{k}\right): e\left(u_{\tau}^{k}\right)-\operatorname{div}\left(\kappa\left|\nabla \alpha_{\tau}^{k}\right|^{p-2} \nabla \alpha_{\tau}^{k}\right) \ni \phi^{\prime}\left(\alpha_{\tau}^{k}\right)
\end{gathered}
$$

considered on $\Omega$ while completed with the corresponding boundary conditions

$$
\begin{aligned}
& \left(\mathbb{C}\left(\alpha_{\tau}^{k}\right) e\left(u_{\tau}^{k}\right)+\mathbb{D}\left(\alpha_{\tau}^{k-1}\right) e\left(v_{\tau}^{k-1 / 2}\right)\right) \cdot \vec{n}=g_{\tau}^{k} \text { and } \\
& \kappa \nabla \alpha_{\tau}^{k} \cdot \vec{n}=0, \quad \text { where } \quad g_{\tau}^{k}:=\int_{(k-1) \tau}^{k \tau} g(t) \mathrm{d} t .
\end{aligned}
$$

It is to be solved iteratively for $k=1, \ldots, T / \tau$ with

$$
u_{\tau}^{0}=u_{0}, \quad v_{\tau}^{0}=v_{0}, \quad \alpha_{\tau}^{0}=\alpha_{0} .
$$

The important property of the underlying energy functionals is convexity. In damage-type problems, the mentioned nonconvexity of the stored energy is suppressed in the time-discrete problems due to the quadratic viscosity potentials, and it suffices to guarantee only some semiconvexity of the stored energy, cf. [40, Remark 8.24] or for damage-type problems [39]. We should emphasize that the attribute of semiconvexity is not automatic and e.g. for $\mathbb{C}(\cdot)$ affine, i.e. for $(e, \alpha) \mapsto \alpha \mathbb{C}^{\prime} e: e$, it does not hold $\mathrm{cf}$. (15) below for $\gamma^{\prime \prime}=0$ and realize that even considering also the term $\phi$ cannot help for $|e| \rightarrow \infty$. Anyhow, here fortunately, we are able to state even a bit strengthened version of the semiconvexity, using the convexification 
by only the e-component but not $\alpha$. This will enable to devise the robust scheme by using the viscosity only in strains but not in $\alpha$, although we will use (and later need) the ratedependent flow rule for $\alpha$ for simplifying some analytical arguments.

Let us first summarize the assumptions needed partly here and especially later:

$$
\begin{aligned}
& \mathbb{C}(\alpha):=\gamma(\alpha) \mathbb{C}_{1} \text { with } \mathbb{C}_{1} \text { positive definite and } \\
& \text { with } \gamma: \mathbb{R} \rightarrow \mathbb{R}^{+} \text {smooth, positive, and strictly convex, } \\
& \mathbb{D}_{0}(\cdot) \text { in }(6 \mathrm{~b}) \text { symmetric positive definite, } \\
& \phi:[0,1] \rightarrow \mathbb{R} \text { continuously differentiable and concave, } \\
& \zeta: \mathbb{R} \rightarrow \mathbb{R} \cup\{+\infty\} \text { convex lower semicontinuous and } \\
& \text { coercive in the sense that } \inf _{v \neq 0} \zeta(v) /|v|^{2}>0 \text {, } \\
& \varrho \in L^{\infty}(\Omega), \operatorname{essinf}_{\Omega} \varrho(\cdot)>0, \quad f \in L^{2}\left(Q ; \mathbb{R}^{d}\right), g \in L^{2}\left(\Sigma ; \mathbb{R}^{d}\right) \text {, } \\
& u_{0} \in H^{1}\left(\Omega ; \mathbb{R}^{d}\right), v_{0} \in L^{2}\left(\Omega ; \mathbb{R}^{d}\right) \text {, and } \alpha_{0} \in W^{1, p}(\Omega) \text {. }
\end{aligned}
$$

Lemma 3.1 (Strenghtened semiconvexity of the stored energy.) Let (13a) hold. Then the function $(e, \alpha) \mapsto \frac{1}{2} \mathbb{C}(\alpha) e: e+\frac{1}{2} K|e|^{2}$ is convex provided $K$ large enough.

Proof. The Hessian of the function in question, i.e.

$$
\left(\begin{array}{cc}
\gamma(\alpha) \mathbb{C}_{1}+K \mathbb{I}, & \gamma^{\prime}(\alpha) \mathbb{C}_{1} e \\
\gamma^{\prime}(\alpha) \mathbb{C}_{1} e & , \frac{1}{2} \gamma^{\prime \prime}(\alpha) \mathbb{C}_{1} e: e
\end{array}\right)=\left(\begin{array}{cc}
\gamma(\alpha) \mathbb{C}_{1}, & 0 \\
0 & , 0
\end{array}\right)+\left(\begin{array}{cc}
K \mathbb{I} & \gamma^{\prime}(\alpha) \mathbb{C}_{1} e \\
\gamma^{\prime}(\alpha) \mathbb{C}_{1} e & \frac{1}{2} \gamma^{\prime \prime}(\alpha) \mathbb{C}_{1} e: e
\end{array}\right)
$$

is to be positive semidefinite for sufficiently big $K$. The former matrix on the right-hand of (14) is surely positive semidefinite. Therefore we are to prove the positive semidefiniteness of the latter one. For any $(\widetilde{e}, \widetilde{\alpha}) \in \mathbb{R}_{\mathrm{sym}}^{d \times d} \times \mathbb{R}$, we can estimate

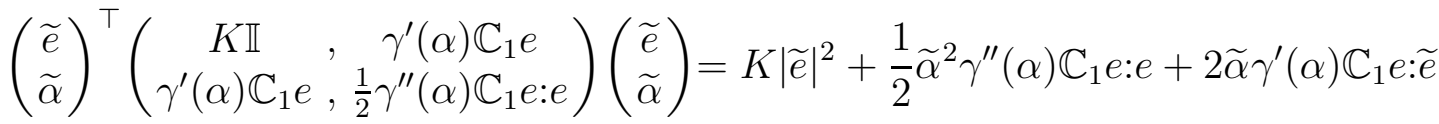

$$
\begin{aligned}
& \geq \widetilde{\alpha}^{2}\left(\frac{1}{2} \gamma^{\prime \prime}(\alpha) \mathbb{C}_{1} e: e-\frac{1}{K} \gamma^{\prime}(\alpha)^{2}\left|\mathbb{C}_{1} e\right|^{2}\right) \\
& \geq \widetilde{\alpha}^{2}\left(\frac{\gamma^{\prime \prime}(\alpha)}{2\left|\mathbb{C}_{1}^{-1}\right|}-\frac{1}{K} \gamma^{\prime}(\alpha)^{2}\left|\mathbb{C}_{1}\right|^{2}\right)|e|^{2} \geq 0,
\end{aligned}
$$

where $1 /\left|\mathbb{C}_{1}^{-1}\right|$ is the positive-definiteness constant of $\mathbb{C}_{1}$, i.e. $1 /\left|\mathbb{C}_{1}^{-1}\right|=\min _{|e|=1} \mathbb{C}_{1} e$ :e. It reveals that, for the last inequality in (15), it suffices to take

$$
K \geq 2\left|\mathbb{C}_{1}\right|^{2}\left|\mathbb{C}_{1}^{-1}\right| \frac{\max \gamma^{\prime}([0,1])^{2}}{\min \gamma^{\prime \prime}([0,1])}
$$

The important attribute behind the scheme (10)-(11) is its variational structure, holding even for any time steps $\tau>0$ without the mentioned convex structure, cf. also [41,43] for the form of (17) if the stored energy were convex: 
Proposition 3.2 (Variational structure.) Let (6) and (13a-e) be valid, $u_{\tau}^{k-1} \in$ $H^{1}\left(\Omega ; \mathbb{R}^{d}\right), v_{\tau}^{k-1} \in L^{2}\left(\Omega ; \mathbb{R}^{d}\right), \alpha_{\tau}^{k-1} \in L^{\infty}(\Omega), 0 \leq \alpha_{\tau}^{k-1} \leq 1$, and let $p>1$. Then the functional

$$
\begin{aligned}
(u, \alpha) \mapsto & \int_{\Omega} \frac{\varrho}{2 \tau}\left|\frac{u-u_{\tau}^{k-1}}{\tau}-v_{\tau}^{k-1}\right|^{2}+\frac{1}{2} \mathbb{C}(\alpha) e(u): e(u)-\phi(\alpha)+\frac{\kappa}{p}|\nabla \alpha|^{p}-f_{\tau}^{k} \cdot u \\
& +\frac{1}{2 \tau} \mathbb{D}\left(\alpha_{\tau}^{k-1}\right) e\left(u-u_{\tau}^{k-1}\right): e\left(u-u_{\tau}^{k-1}\right)+\tau \zeta\left(\frac{\alpha-\alpha_{\tau}^{k-1}}{\tau}\right) \mathrm{d} x-\int_{\Gamma} g_{\tau}^{k} \cdot u \mathrm{~d} S
\end{aligned}
$$

is weakly lower semicontinuous on $H^{1}\left(\Omega ; \mathbb{R}^{d}\right) \times H^{1}(\Omega)$. For any $\left(u_{\tau}^{k}, v_{\tau}^{k}, \alpha_{\tau}^{k}\right) \in H^{1}\left(\Omega ; \mathbb{R}^{d}\right) \times$ $L^{2}\left(\Omega ; \mathbb{R}^{d}\right) \times W^{1, p}(\Omega)$ solving (in the usual weak sense) the boundary value problem (10) -(11), the couple $\left(u_{\tau}^{k}, \alpha_{\tau}^{k}\right)$ is a critical point of this functional. Also, conversely, any critical point $(u, \alpha)$ of (17) gives a weak solution $\left(u_{\tau}^{k}, v_{\tau}^{k}, \alpha_{\tau}^{k}\right)$ to (10)-(11) when putting

$$
u_{\tau}^{k}=u, \quad v_{\tau}^{k}=\frac{2}{\tau}\left(u_{\tau}^{k}-u_{\tau}^{k-1}\right)-v_{\tau}^{k-1}, \quad \alpha_{\tau}^{k}=\alpha .
$$

In particular, such solutions do exist. Moreover, the potential (17) is strictly convex provided $\tau$ is sufficiently small, namely

$$
\tau \leq \tau_{0}=\frac{\min \gamma^{\prime \prime}([0,1])}{2\left|\mathbb{D}_{0}^{-1}\right|\left|\mathbb{C}_{1}\right|^{2}\left|\mathbb{C}_{1}^{-1}\right| \max \gamma^{\prime}([0,1])^{2}} \quad \text { with } \mathbb{D}_{0}:=\mathbb{D}(0) \text { as in (하) }
$$

Proof. The system (10) does not satisfy the usual symmetry condition and thus does not have any potential, but eliminating $v_{\tau}^{k}$ by substituting $v_{\tau}^{k}$ as in (18), we obtain a potential for the couple $\left(u_{\tau}^{k}, \alpha_{\tau}^{k}\right)$, and it is not difficult to identify its form as (17).

For the convexity, we use Lemma 3.1 for the $\mathbb{C}$-term, which is the only nonconvex term in (17), with $K$ replaced by $\varepsilon / \tau$ with $\varepsilon=1 /\left|\mathbb{D}_{0}^{-1}\right|$ the positive-definiteness constant of $\mathbb{D}_{0}$, i.e. $\mathbb{D}_{0} e: e \geq \varepsilon|e|^{2}$. Then (16) yields (19). The strict convexity of the overall functional (17) then follows from the strict convexity of the $\varrho$ - and $\kappa$-terms in (17).

Let us note that, as the physical dimension of $\mathbb{C}_{1}$ is $\mathrm{Pa}$ and of $\mathbb{D}_{0}$ is Pas, the right-hand side of (19) has the physical dimension indeed seconds, as expected.

In particular, for sufficiently small time steps, the formula (10)-(11) possesses just one weak solution.

Remark 3.3 (Insufficiency of viscosity in damage.) It is notable that inventing some viscosity into the damage flow-rule (as sometimes considered in engineering literature [23, $28,31,52])$ cannot stabilize the monolithic scheme. This is because the function $(e, \alpha) \mapsto$ $\frac{1}{2} \mathbb{C}(\alpha) e: e+\frac{1}{2} K \alpha^{2}$ is not convex in general, no matter how $K$ is big. This can be seen even for $d=1$ by analysing the positive definiteness of the corresponding Hessian, whose determinant, in contrast to (15), would then contain the factor $\frac{1}{2} \gamma^{\prime \prime}(\alpha) \mathbb{C}_{1} e: e+K-\gamma^{\prime}(\alpha)^{2} e \mathbb{C}_{1} \mathbb{C}^{-1}(0) \mathbb{C}_{1} e$ which, for $|e| \rightarrow \infty$, eliminates the influence of $K$ and the positive definiteness is surely corrupted if $\mathbb{C}(0)$ is small (as usually accepted as a modelling assumption).

Remark 3.4 (Fully implicit scheme.) Note that the scheme (10)-(11) is truly fully implicit only if $\chi_{\mathrm{R}}=0$ because we took the viscous moduli "delayed", i.e. $\chi_{\mathrm{R}} \mathbb{C}\left(\alpha_{\tau}^{k-1}\right)$, instead of $\chi_{\mathrm{R}} \mathbb{C}\left(\alpha_{\tau}^{k}\right)$ which would be a considerable alternative but would corrupt the variational structure which was proved in Proposition 3.2 and which may be advantageous for some optimization algorithms. Yet, e.g. the mentioned Newton-Raphson may not rely on existence of some potentials and then such a fully implicit scheme can be considered, even for $p>1$ since the last term in (29) and its estimation (35) below would be avoided. 


\section{A-priori estimates and convergence for $\tau \rightarrow 0$}

Thorough the whole paper, we will use the standard notation for the Lebesgue $L^{p}$-spaces and $W^{k, p}$ for Sobolev spaces whose $k$-th distributional derivatives are in $L^{p}$-spaces. We will also use the abbreviation $H^{k}=W^{k, 2}$. In the vectorial case, we will write $L^{p}\left(\Omega ; \mathbb{R}^{n}\right) \cong L^{p}(\Omega)^{n}$ and $W^{1, p}\left(\Omega ; \mathbb{R}^{n}\right) \cong W^{1, p}(\Omega)^{n}$. On the time interval $I=[0, T]$, we consider the Bochner spaces $L^{p}(I ; X)$ of Bochner measurable mappings $I \rightarrow X$ whose norm is in $L^{p}(I)$, with $X$ being a Banach space, while $C_{\mathrm{w}}(I ; X)$ will denote the Banach space of weakly continuous mappings $I \rightarrow X$. We recall using the notation $Q=I \times \Omega$ and $\Sigma=I \times \Gamma$.

Considering a fixed (sufficiently small to make (17) convex) time step $\tau>0$ and $\left\{u_{\tau}^{k}\right\}_{k=0, \ldots, T / \tau}$ with $T / \tau \in \mathbb{N}$, we define the piecewise-constant and the piecewise affine interpolants respectively by

$$
\begin{array}{ll}
\bar{u}_{\tau}(t)=u_{\tau}^{k}, \quad \underline{u}_{\tau}(t)=u_{\tau}^{k-1}, \quad \underline{\bar{u}}_{\tau}(t)=\frac{1}{2} u_{\tau}^{k}+\frac{1}{2} u_{\tau}^{k-1}, \quad \text { and } \\
u_{\tau}(t)=\frac{t-(k-1) \tau}{\tau} u_{\tau}^{k}+\frac{k \tau-t}{\tau} u_{\tau}^{k-1} & \text { for }(k-1) \tau<t \leq k \tau .
\end{array}
$$

For $\left\{\left(v_{\tau}^{k}, \alpha_{\tau}^{k}, f_{\tau}^{k}, g_{\tau}^{k}\right)\right\}_{k=0, \ldots, T / \tau}$, the similar meaning has also $\underline{\bar{v}}_{\tau}, \alpha_{\tau}, \bar{\alpha}_{\tau}, \bar{f}_{\tau}, \bar{g}_{\tau}$, etc. In terms of these interpolants, the scheme (10)-(11) can be written "more compactly" as:

$$
\begin{aligned}
& \dot{u}_{\tau}=\underline{\bar{v}}_{\tau} \text { and } \varrho \dot{v}_{\tau}-\operatorname{div}\left(\mathbb{C}\left(\bar{\alpha}_{\tau}\right) e\left(\bar{u}_{\tau}\right)+\mathbb{D}\left(\underline{\alpha}_{\tau}\right) e\left(\underline{\underline{v}}_{\tau}\right)\right)=\bar{f}_{\tau}, \\
& \partial \zeta\left(\dot{\alpha}_{\tau}\right)+\frac{1}{2} \mathbb{C}^{\prime}\left(\bar{\alpha}_{\tau}\right) e\left(\bar{u}_{\tau}\right): e\left(\bar{u}_{\tau}\right)-\operatorname{div}\left(\kappa\left|\nabla \bar{\alpha}_{\tau}\right|^{p-2} \nabla \bar{\alpha}_{\tau}\right) \ni \phi^{\prime}\left(\bar{\alpha}_{\tau}\right)
\end{aligned}
$$

with the boundary conditions

$$
\left(\mathbb{C}\left(\bar{\alpha}_{\tau}\right) e\left(\bar{u}_{\tau}\right)+\mathbb{D}\left(\underline{\alpha}_{\tau}\right) e\left(\underline{\bar{v}}_{\tau}\right)\right) \vec{n}=\bar{g}_{\tau} \quad \text { and } \quad \nabla \bar{\alpha}_{\tau} \cdot \vec{n}=0 \quad \text { on } \Sigma .
$$

Proposition 4.1 (Discrete energetics.) Let the assumptions (6) and (13) be fulfilled and $p>1$, and let $\tau_{0}>0$ be taken from (19). Then the analog of (7) as an inequality

$$
\begin{aligned}
& \mathscr{T}\left(\dot{u}_{\tau}(t)\right)+\mathscr{E}\left(u_{\tau}(t), \alpha_{\tau}(t)\right)+\left(1-\sqrt{\frac{\tau}{\tau_{0}}}\right) \int_{0}^{t} \Xi\left(\underline{\alpha}_{\tau} ; \dot{u}_{\tau}, \dot{\alpha}_{\tau}\right) \mathrm{d} t \\
& \leq \mathscr{T}\left(v_{0}\right)+\mathscr{E}\left(u_{0}, \alpha_{0}\right)+\int_{0}^{t} \mathscr{F}\left(\bar{t}_{\tau}, \dot{u}_{\tau}\right) \mathrm{d} t
\end{aligned}
$$

holds for any $t=k \tau$ with $k=1, \ldots, T / \tau$, with $\bar{t}_{\tau}(t):=k \tau$ for $t \in((k-1 \tau, k \tau)$, provided $\tau>0$ is sufficiently small satisfying (19).

Proof. To pursue the physical energy estimates, we test the first equation in (21a) by $\varrho \dot{v}_{\tau}$, the second equation in (21a) by $\dot{u}_{\tau}=\underline{\bar{v}}_{\tau}$, and (21b) by $\dot{\alpha}_{\tau}$. The first test leads, by the binomial formula, to the equality

$$
\begin{aligned}
\int_{0}^{T} \varrho \dot{v}_{\tau} \cdot \dot{u}_{\tau} \mathrm{d} t & =\int_{0}^{T} \varrho \dot{v}_{\tau} \cdot \bar{v}_{\tau} \mathrm{d} t=\sum_{k=1}^{T / \tau} \varrho \frac{v_{\tau}^{k}-v_{\tau}^{k-1}}{\tau} \cdot \frac{v_{\tau}^{k}+v_{\tau}^{k-1}}{2} \\
& =\sum_{k=1}^{T / \tau} \frac{\varrho}{2}\left|v_{\tau}^{k}\right|^{2}-\frac{\varrho}{2}\left|v_{\tau}^{k-1}\right|^{2}=\frac{\varrho}{2}\left|v_{\tau}(T)\right|^{2}-\frac{\varrho}{2}\left|v_{0}\right|^{2}
\end{aligned}
$$


a.e. on $\Omega$. For the second and the third mentioned tests, we use the notation $(7 \mathrm{~b}, \mathrm{c})$ to write $(10 \mathrm{~b}, \mathrm{c})-(11)$ shortly in an abstract way as

$$
\mathscr{T}^{\prime} \frac{v_{\tau}^{k}-v_{\tau}^{k-1}}{\tau}+\mathscr{E}^{\prime}\left(u_{\tau}^{k}, \alpha_{\tau}^{k}\right)+\mathscr{D}_{(v, \alpha)}^{\prime}\left(\alpha^{k-1} ; \frac{u_{\tau}^{k}-u_{\tau}^{k-1}}{\tau}, \frac{\alpha_{\tau}^{k}-\alpha_{\tau}^{k-1}}{\tau}\right) \ni \mathscr{F}_{\tau}^{k}
$$

with $\mathscr{F}_{\tau}^{k}$ as in (1d) but with $f_{\tau}^{k}$ and $g_{\tau}^{k}$ from (10b)- 11b), and we test it by $\left(u_{\tau}^{k}, \alpha_{\tau}^{k}\right)-\left(u_{\tau}^{k-1}, \alpha_{\tau}^{k-1}\right)$. The first term was already done in (23). The second and the third terms can be estimated by using the semiconvexity proved in Lemma 3.1, cf. also [40, Remark 8.24] for this estimation technique. Here, denoting $\mathscr{D}_{0}(v):=\int_{\Omega} \frac{1}{2} \mathbb{D}_{0} e(v): e(v) \mathrm{d} x$ and $\Xi_{0}(v):=\int_{\Omega} \mathbb{D}_{0} e(v): e(v) \mathrm{d} x$ and taking some reference time step $\tau_{0}>0$, it gives

$$
\begin{aligned}
\left\langle\mathscr{D}_{(v, \alpha)}^{\prime}\left(\alpha^{k-1} ; \frac{u_{\tau}^{k}-u_{\tau}^{k-1}}{\tau}, \frac{\alpha_{\tau}^{k}-\alpha_{\tau}^{k-1}}{\tau}\right)+\mathscr{E}^{\prime}\left(u_{\tau}^{k}, \alpha_{\tau}^{k}\right), \frac{\left(u_{\tau}^{k}, \alpha_{\tau}^{k}\right)-\left(u_{\tau}^{k-1}, \alpha_{\tau}^{k-1}\right)}{\tau}\right\rangle \\
\geq \Xi_{0}\left(\frac{u_{\tau}^{k}-u_{\tau}^{k-1}}{\tau}\right)+\left\langle\mathscr{E}^{\prime}\left(u_{\tau}^{k}, \alpha_{\tau}^{k}\right), \frac{\left(u_{\tau}^{k}, \alpha_{\tau}^{k}\right)-\left(u_{\tau}^{k-1}, \alpha_{\tau}^{k-1}\right)}{\tau}\right\rangle \\
=\left\langle\mathscr{D}_{0}^{\prime}\left(\frac{u_{\tau}^{k}-u_{\tau}^{k-1}}{\tau}\right)+\mathscr{E}^{\prime}\left(u_{\tau}^{k}, \alpha_{\tau}^{k}\right), \frac{\left(u_{\tau}^{k}, \alpha_{\tau}^{k}\right)-\left(u_{\tau}^{k-1}, \alpha_{\tau}^{k-1}\right)}{\tau}\right\rangle \\
=\left\langle\frac{1}{\sqrt{\tau_{0} \tau}} \mathscr{D}_{0}^{\prime}\left(u_{\tau}^{k}\right)+\mathscr{E}^{\prime}\left(u_{\tau}^{k}, \alpha_{\tau}^{k}\right), \frac{\left(u_{\tau}^{k}, \alpha_{\tau}^{k}\right)-\left(u_{\tau}^{k-1}, \alpha_{\tau}^{k-1}\right)}{\tau}\right\rangle \\
\quad-\left\langle\frac{1}{\sqrt{\tau_{0} \tau}} \mathscr{D}_{0}^{\prime}\left(u_{\tau}^{k-1}\right), \frac{u_{\tau}^{k}-u_{\tau}^{k-1}}{\tau}\right\rangle+\left(1-\sqrt{\frac{\tau}{\tau_{0}}}\right) \Xi_{0}\left(\frac{u_{\tau}^{k}-u_{\tau}^{k-1}}{\tau}\right) \\
\geq \frac{1}{\tau}\left(\frac{1}{\sqrt{\tau_{0} \tau}} \mathscr{D}_{0}\left(u_{\tau}^{k}\right)+\mathscr{E}\left(u_{\tau}^{k}, \alpha_{\tau}^{k}\right)-\frac{1}{\sqrt{\tau_{0} \tau}} \mathscr{D}_{0}\left(u_{\tau}^{k-1}\right)-\mathscr{E}\left(u_{\tau}^{k-1}, \alpha_{\tau}^{k-1}\right)\right) \\
\quad-\left\langle\frac{1}{\sqrt{\tau_{0} \tau}} \mathscr{D}_{0}^{\prime}\left(u_{\tau}^{k-1}\right), \frac{u_{\tau}^{k}-u_{\tau}^{k-1}}{\tau}\right\rangle+\left(1-\sqrt{\frac{\tau}{\tau_{0}}}\right) \Xi_{0}\left(\frac{u_{\tau}^{k}-u_{\tau}^{k-1}}{\tau}\right) \\
=\frac{\mathscr{E}\left(u_{\tau}^{k}, \alpha_{\tau}^{k}\right)-\mathscr{E}_{\tau}\left(u_{\tau}^{k-1}, \alpha_{\tau}^{k-1}\right)}{\tau}+\left(1-\sqrt{\frac{\tau}{\tau_{0}}}\right) \Xi_{0}\left(\frac{u_{\tau}^{k}-u_{\tau}^{k-1}}{\tau}\right) .
\end{aligned}
$$

Here we use convexity of $\mathscr{E}+\mathscr{D}_{0} / \sqrt{\tau_{0} \tau}$ provided $\tau>0$ is small enough, which follows from the convexity of $(e, \alpha) \mapsto \mathbb{C}(\alpha) e: e+\mathbb{D}_{0} e: e / \sqrt{\tau_{0} \tau}$ for $\tau \leq \tau_{0}$ with $\tau_{0}$ from (19).

The resting contribution to the dissipation rate $\Xi$ is even with the factor 1 . Altogether, (22) is proved.

Proposition 4.2 (Numerical stability - a-priori estimates.) Let the assumptions of Proposition 4.1 be fulfilled, and let now $\tau \leq \tau_{0} / 2$ with $\tau_{0}$ from (19). Then, the following a-priori estimates hold with $C$ independent of $\tau$ :

$$
\begin{aligned}
& \left\|u_{\tau}\right\|_{H^{1}\left(I ; H^{1}\left(\Omega ; \mathbb{R}^{d}\right)\right)} \leq C \quad \text { and } \quad\left\|u_{\tau}\right\|_{W^{1, \infty}\left(I ; L^{2}\left(\Omega ; \mathbb{R}^{d}\right)\right)} \leq C, \\
& \left\|v_{\tau}\right\|_{L^{2}\left(I ; H^{1}\left(\Omega ; \mathbb{R}^{d}\right)\right)} \leq C \quad \text { and } \quad\left\|u_{\tau}\right\|_{L^{\infty}\left(I ; L^{2}\left(\Omega ; \mathbb{R}^{d}\right)\right)} \leq C, \\
& \left\|\alpha_{\tau}\right\|_{L^{\infty}\left(I ; W^{1, p}(\Omega)\right)} \leq C \quad \text { and } \quad\left\|\alpha_{\tau}\right\|_{H^{1}\left(I ; L^{2}(\Omega)\right)} \leq C,
\end{aligned}
$$

and, if in addition $\varrho \in W^{1, p}(\Omega)$ with $p>2$ if $d=2$ or $p=3$ if $d=3$, then also

$$
\left\|\sqrt{\varrho} \dot{v}_{\tau}\right\|_{L^{2}\left(I ; H^{1}\left(\Omega ; \mathbb{R}^{d}\right)^{*}\right)} \leq C .
$$


Proof. We have (22) with the factor $(1-\sqrt{1 / 2})$ in front of the $\Xi$-term at disposal. The righthand side of (22) estimated from above by $\int_{0}^{t} C\|f(t)\|_{L^{2}\left(\Omega ; \mathbb{R}^{d}\right)}^{2}+C\|g(t)\|_{L^{2}\left(\Omega ; \mathbb{R}^{d}\right)}^{2}+\frac{1}{4} \Xi_{0}\left(\dot{u}_{\tau}(t)\right) \mathrm{d} t$ with some $C$ large enough, and then $\frac{1}{4} \Xi_{0}\left(\dot{u}_{\tau}\right)$ can be absorbed in the dissipation term in the left-hand side. From the coercivity of $\mathscr{T}, \mathscr{E}$, and $\Xi(\alpha ; \cdot, \cdot)$, we eventually obtain (25).

The estimate (25d) can be proved by a comparison $\varrho \dot{v}_{\tau}=\operatorname{div}\left(\mathbb{C}\left(\bar{\alpha}_{\tau}\right) e\left(\bar{u}_{\tau}\right)+\mathbb{D}\left(\underline{\alpha}_{\tau}\right) e\left(\underline{\bar{v}}_{\tau}\right)\right)+$ $\bar{f}_{\tau}$, cf. (21a). More in detail, when testing it by $z \in L^{2}\left(I ; H^{1}\left(\Omega ; \mathbb{R}^{d}\right)\right)$ and using also the boundary conditions (21c), one arrives to

$$
\begin{aligned}
\left\|\sqrt{\varrho} \dot{v}_{\tau}\right\|_{L^{2}\left(I ; H^{1}\left(\Omega ; \mathbb{R}^{d}\right)^{*}\right)}= & \sup _{\|z\|_{L^{2}\left(I ; H^{1}\left(\Omega ; \mathbb{R}^{d}\right)\right)} \leq 1}\left(\int_{\Sigma} \bar{g}_{\tau} \cdot \frac{z}{\sqrt{\varrho}} \mathrm{d} S \mathrm{~d} t\right. \\
& \left.\quad+\int_{Q} \bar{f}_{\tau} \cdot \frac{z}{\sqrt{\varrho}}-\left(\mathbb{C}\left(\bar{\alpha}_{\tau}\right) e\left(\bar{u}_{\tau}\right)+\mathbb{D}\left(\underline{\alpha}_{\tau}\right) e\left(\underline{\underline{v}}_{\tau}\right)\right): \nabla \frac{z}{\sqrt{\varrho}} \mathrm{d} x \mathrm{~d} t\right),
\end{aligned}
$$

from which (25d) follows by the Hölder inequality and by using the already obtained estimates (25a,b), cf. also [24, Sect.6.4].

The concept of weak solutions to the original continuous problem (44) is a bit delicate because the damage driving force (or a "pressure") $\frac{1}{2} \mathbb{C}^{\prime}(\alpha) e(u): e(u)$ is bounded only in $L^{1}(Q)$ and testing it by $\dot{\alpha} \in L^{2}(Q)$ as occurs in (5) is not legitimate. To cope with this problem, this possibly not-integrable term can be substituted by

$$
\begin{aligned}
\int_{Q} \frac{1}{2} \mathbb{C}^{\prime}(\alpha) e(u): e(u) \dot{\alpha} \mathrm{d} x \mathrm{~d} t= & \int_{\Omega} \frac{1}{2} \mathbb{C}(\alpha(T)) e(u(T)): e(u(T)) \mathrm{d} x \\
& -\int_{Q} \mathbb{C}(\alpha) e(u): e(\dot{u}) \mathrm{d} x \mathrm{~d} t-\int_{\Omega} \frac{1}{2} \mathbb{C}(\alpha)_{0} e\left(u_{0}\right): e\left(u_{0}\right) \mathrm{d} x .
\end{aligned}
$$

Similar note is about the gradient term $\operatorname{div}\left(\kappa|\nabla \alpha|^{p-2} \nabla \alpha\right)$. This last term can be just integrated by parts, i.e. $\int_{Q} \operatorname{div}\left(\kappa|\nabla \alpha|^{p-2} \nabla \alpha\right) \dot{\alpha} \mathrm{d} x \mathrm{~d} t=\int_{\Omega} \frac{\kappa}{p}\left|\nabla \alpha_{0}\right|^{p}-\frac{\kappa}{p}|\nabla \alpha(T)|^{p} \mathrm{~d} x$. Thus we obtain the following:

Definition 4.3 (Weak solutions.) The pair $(u, \alpha) \in H^{1}\left(I ; H^{1}\left(\Omega ; \mathbb{R}^{d}\right)\right) \times C_{\mathrm{w}}\left(I ; W^{1, p}(\Omega)\right)$ is a weak solution to the initial-boundary-value problem (9) if $\dot{u} \in C_{\mathrm{w}}\left(I ; L^{2}\left(\Omega ; \mathbb{R}^{d}\right)\right), \zeta(\dot{\alpha}) \in$ $L^{1}(Q), 0 \leq \alpha \leq 1$ a.e. on $Q$, and

$$
\begin{aligned}
& \forall v \in L^{2}\left(I ; H^{1}\left(\Omega ; \mathbb{R}^{d}\right)\right) \cap H^{1}\left(I ; L^{2}\left(\Omega ; \mathbb{R}^{d}\right)\right),\left.\quad v\right|_{t=T}=0: \\
& \int_{Q} \mathbb{D}(\alpha) e(\dot{u})+\mathbb{C}(\alpha) e(u): e(v)-\varrho \dot{u} \cdot \dot{v} \mathrm{~d} x \mathrm{~d} t \\
& \quad=\int_{\Omega} \varrho v_{0} \cdot v(0, \cdot) \mathrm{d} x+\int_{Q} f \cdot v \mathrm{~d} x \mathrm{~d} t+\int_{\Sigma} g \cdot v \mathrm{~d} S \mathrm{~d} t,
\end{aligned}
$$

$$
\forall z \in L^{1}\left(I ; W^{1, p}(\Omega)\right) \cap L^{\infty}(Q):
$$

$$
\begin{aligned}
& \int_{Q}\left(\frac{1}{2} \mathbb{C}^{\prime}(\alpha) e(u): e(u)-\phi^{\prime}(\alpha)\right) z+\kappa|\nabla \alpha|^{p-2} \nabla \alpha \cdot \nabla z+\zeta(z) \mathrm{d} x \mathrm{~d} t \\
& \geq \int_{Q} \zeta(\dot{\alpha})+ \mathbb{C}(\alpha) e(u): e(\dot{u}) \mathrm{d} x \mathrm{~d} t+\int_{\Omega}\left(\frac{1}{2} \mathbb{C}(\alpha(T)) e(u(T)): e(u(T))-\phi(\alpha(T))\right. \\
&+\left.\frac{\kappa}{p}|\nabla \alpha(T)|^{p}-\frac{1}{2} \mathbb{C}\left(\alpha_{0}\right) e\left(u_{0}\right): e\left(u_{0}\right)+\phi\left(\alpha_{0}\right)-\frac{\kappa}{p}\left|\nabla \alpha_{0}\right|^{p}\right) \mathrm{d} x
\end{aligned}
$$


and if also the resting initial conditions hold, i.e. $\left.u\right|_{t=0}=u_{0}$ and $\left.\alpha\right|_{t=0}=\alpha_{0}$ (while $\left.\dot{u}\right|_{t=0}=v_{0}$ is already involved in (26a)).

Proposition 4.4 (Convergence for $\tau \rightarrow 0$.) Let the assumptions of Proposition 4.1 be fulfilled now with $p>d$. Then there exists a selected subsequence and its limit $(u, \alpha)$ such that

$$
\begin{aligned}
& u_{\tau} \rightarrow u \text { in } H^{1}\left(I ; H^{1}\left(\Omega ; \mathbb{R}^{d}\right)\right)(\text { weakly }) \text { and } u_{\tau} *^{*} u \text { in } L^{\infty}\left(I ; L^{2}\left(\Omega ; \mathbb{R}^{d}\right)\right)(\text { weakly*), } \\
& v_{\tau} \rightarrow v \text { in } L^{2}\left(I ; H^{1}\left(\Omega ; \mathbb{R}^{d}\right)\right)(\text { weakly }) \text { and } v=\dot{u}, \\
& \alpha_{\tau}{ }^{*} \alpha \text { in } L^{\infty}\left(I ; W^{1, p}(\Omega)\right)(\text { weakly } *) \text { and } \alpha_{\tau} \rightarrow \alpha \text { in } H^{1}\left(I ; L^{2}(\Omega)\right)(\text { weakly })
\end{aligned}
$$

for $\tau \rightarrow 0$. Moreover, every such a subsequence converges also as

$$
u_{\tau} \rightarrow u \text { in } H^{1}\left(I ; H^{1}\left(\Omega ; \mathbb{R}^{d}\right)\right) \text { (strongly), }
$$

and every such a limit $(u, \alpha)$ is a weak solution to the initial-boundary-value problem (9) according Definition 4.3 .

Proof. After selecting a subsequence converging weakly* in the topologies indicated in (25) and using the Aubin-Lions theorem for the damage and then continuity of the superposition operator induced by $\mathbb{C}(\cdot)$, we can pass to the limit first in the semilinear force-equlibrium equation, obtaining (9a,b,d,e) in the weak form (26a).

For the damage flow rule, we need the strong convergence (27d), however. The strategy for this strong convergence is to use the new variable $w=u+\chi_{\mathrm{R}} v$ as in [25], and denote the piecewise affine and the piecewise constant interpolants respectively as

$$
w_{\tau}=u_{\tau}+\chi_{\mathrm{R}} v_{\tau} \quad \text { and } \quad \bar{w}_{\tau}=\bar{u}_{\tau}+\chi_{\mathrm{R}} \dot{u}_{\tau}=\bar{u}_{\tau}+\chi_{\mathrm{R}} \underline{\bar{v}}_{\tau} .
$$

Then we can write the time-discrete approximation of the force equilibrium (21a) equivalently as

$$
\frac{\varrho}{\chi_{\mathrm{R}}} \dot{w}_{\tau}-\operatorname{div}\left(\mathbb{D}_{0} e\left(\underline{\underline{v}}_{\tau}\right)+\mathbb{C}\left(\bar{\alpha}_{\tau}\right) e\left(\bar{w}_{\tau}\right)\right)=\bar{f}_{\tau}+\frac{\varrho}{\chi_{\mathrm{R}}} \dot{u}_{\tau}+\operatorname{div}\left(\left(\mathbb{C}\left(\bar{\alpha}_{\tau}\right)-\mathbb{C}\left(\underline{\alpha}_{\tau}\right)\right) e\left(\dot{u}_{\tau}\right)\right)
$$

accompanied with the initial/boundary conditions (9k,d) and similarly also the mentioned limit force equilibrium can be written in such a form, namely

$$
\frac{\varrho}{\chi_{\mathrm{R}}} \dot{w}-\operatorname{div}\left(\mathbb{D}_{0} e(v)+\mathbb{C}(\alpha) e(w)\right)=f+\frac{\varrho}{\chi_{\mathrm{R}}} \dot{u}
$$

accompanied with the initial/boundary conditions $\left(\mathbb{D}_{0} e(v)+\mathbb{C}(\alpha) e(w)\right) \vec{n}=g$ and $w(0)=$ $w_{0}:=u_{0}+\chi_{\mathrm{R}} v_{0}$. An important fact is that, by comparison, we also know $\dot{w} \in$ $L^{2}\left(I ; H^{1}\left(\Omega ; \mathbb{R}^{d}\right)^{*}\right)$ because $\operatorname{div}\left(\mathbb{D}_{0} v\right)$ and $\operatorname{div}(\mathbb{C}(\alpha) e(w))$ belong to $L^{2}\left(I ; H^{1}\left(\Omega ; \mathbb{R}^{d}\right)^{*}\right)$ so that $\dot{w}$ and $\operatorname{div}\left(\mathbb{D}_{0} v\right)$ and $\operatorname{div}(\mathbb{C}(\alpha) e(w))$ are in duality with $w \in L^{2}\left(I ; H^{1}\left(\Omega ; \mathbb{R}^{d}\right)\right)$. Thus, testing (30) by $w$ and integrating over $Q$, we obtain the conservation of mechanical energy in the form

$$
\begin{aligned}
& \int_{\Omega} \frac{\varrho}{2 \chi_{\mathrm{R}}}|w(T)|^{2}+\frac{1}{2} \mathbb{D}_{0} e(u(T)): e(u(T)) \mathrm{d} x+\int_{Q} \mathbb{D}_{0} \chi_{\mathrm{R}} e(v): e(v)+\mathbb{C}(\alpha) e(w): e(w) \mathrm{d} x \mathrm{~d} t \\
& =\int_{\Omega} \frac{\varrho}{2 \chi_{\mathrm{R}}}\left|w_{0}\right|^{2}+\frac{1}{2} \mathbb{D}_{0} e\left(u_{0}\right): e\left(u_{0}\right) \mathrm{d} x+\int_{Q}\left(f+\frac{\varrho}{\chi_{\mathrm{R}}} \dot{u}\right) \cdot w \mathrm{~d} x \mathrm{~d} t+\int_{\Sigma} g \cdot w \mathrm{~d} S \mathrm{~d} t .
\end{aligned}
$$


We further test (30) by $\bar{w}_{\tau}$, and integrate over the time interval $[0, k \tau]$ with $k=1, \ldots, T / \tau$. We use

$$
\begin{aligned}
& \int_{Q} \varrho \dot{w}_{\tau} \cdot \bar{w}_{\tau} \mathrm{d} x \mathrm{~d} t=\int_{Q} \varrho\left(\dot{u}_{\tau}+\chi_{\mathrm{R}} \dot{v}_{\tau}\right) \cdot\left(\bar{u}_{\tau}+\chi_{\mathrm{R}} \overline{\underline{v}}_{\tau}\right) \mathrm{d} x \mathrm{~d} t \\
& =\int_{Q} \varrho\left(\dot{u}_{\tau}+\chi_{\mathrm{R}} \dot{v}_{\tau}\right) \cdot\left(\underline{\bar{u}}_{\tau}+\chi_{\mathrm{R}} \overline{\underline{v}}_{\tau}\right)+\left(\dot{u}_{\tau}+\chi_{\mathrm{R}} \dot{v}_{\tau}\right) \cdot\left(\bar{u}_{\tau}-\underline{\bar{u}}_{\tau}\right) \mathrm{d} x \mathrm{~d} t \\
& =\int_{\Omega} \frac{\varrho}{2}\left|u_{\tau}(T)+\chi_{\mathrm{R}} v_{\tau}(T)\right|^{2}-\frac{\varrho}{2}\left|u_{0}+\chi_{\mathrm{R}} v_{0}\right|^{2} \mathrm{~d} x+\underbrace{\frac{\tau}{2} \int_{Q} \varrho\left(\dot{u}_{\tau}+\chi_{\mathrm{R}} \dot{v}_{\tau}\right) \cdot \dot{u}_{\tau} \mathrm{d} x \mathrm{~d} t}_{=\mathscr{O}(\tau)}
\end{aligned}
$$

where we used also (23) and the estimate (25a) for the last integral. Further, we use

$$
\begin{aligned}
& \int_{0}^{T} \mathbb{D}_{0} e\left(\underline{\bar{v}}_{\tau}\right): e\left(\bar{w}_{\tau}\right) \mathrm{d} t=\int_{0}^{T} \chi_{\mathrm{R}} \mathbb{D}_{0} e\left(\underline{\bar{v}}_{\tau}\right): e\left(\underline{\bar{v}}_{\tau}\right)+\mathbb{D}_{0} e\left(\dot{u}_{\tau}\right): e\left(\bar{u}_{\tau}\right) \mathrm{d} t \\
& \quad \geq \int_{0}^{T} \chi_{\mathrm{R}} \mathbb{D}_{0} e\left(\underline{\bar{v}}_{\tau}\right): e\left(\underline{\underline{v}}_{\tau}\right) \mathrm{d} t+\frac{1}{2} \mathbb{D}_{0} e\left(u_{\tau}(T)\right): e\left(u_{\tau}(T)\right)-\frac{1}{2} \mathbb{D}_{0} e\left(u_{0}\right): e\left(u_{0}\right)
\end{aligned}
$$

a.e. on $\Omega$. We thus obtain the estimate

$$
\begin{aligned}
\limsup _{\tau \rightarrow 0} \int_{Q} \chi_{\mathrm{R}} \mathbb{D}_{0} e\left(\underline{\underline{v}}_{\tau}\right): e\left(\underline{\bar{v}}_{\tau}\right) \mathrm{d} x \mathrm{~d} t \leq \int_{\Omega} \frac{\varrho}{2 \chi_{\mathrm{R}}}\left|u_{0}+\chi_{\mathrm{R}} v_{0}\right|^{2}+\frac{1}{2} \mathbb{D}_{0} e\left(u_{0}\right): e\left(u_{0}\right) \mathrm{d} x \\
-\liminf _{\tau \rightarrow 0}\left(\int_{Q} \mathbb{C}\left(\bar{\alpha}_{\tau}\right) e\left(\bar{w}_{\tau}\right): e\left(\bar{w}_{\tau}\right) \mathrm{d} x \mathrm{~d} t\right. \\
\left.\quad+\int_{\Omega} \frac{\varrho}{2 \chi_{\mathrm{R}}}\left|u_{\tau}(T)+\chi_{\mathrm{R}} v_{\tau}(T)\right|^{2}+\frac{1}{2} \mathbb{D}_{0} e\left(u_{\tau}(T)\right): e\left(u_{\tau}(T)\right) \mathrm{d} x\right) \\
+\lim _{\tau \rightarrow 0}\left(\int_{Q} \bar{f}_{\tau} \cdot \bar{w}_{\tau}+\left(\mathbb{C}\left(\underline{\alpha}_{\tau}\right)-\mathbb{C}\left(\bar{\alpha}_{\tau}\right) e\left(\dot{u}_{\tau}\right): e\left(\bar{w}_{\tau}\right) \mathrm{d} x \mathrm{~d} t+\int_{\Sigma} \bar{g}_{\tau} \cdot \bar{w}_{\tau} \mathrm{d} S \mathrm{~d} t+\mathscr{O}(\tau)\right)\right. \\
\leq \int_{\Omega} \frac{\varrho}{2 \chi_{\mathrm{R}}}\left|u_{0}+\chi_{\mathrm{R}} v_{0}\right|^{2}+\frac{1}{2} \mathbb{D}_{0} e\left(u_{0}\right): e\left(u_{0}\right) \mathrm{d} x+\int_{Q} f \cdot w-\mathbb{C}(\alpha) e(w): e(w) \mathrm{d} x \mathrm{~d} t \\
\quad-\int_{\Omega} \frac{\varrho}{2 \chi_{\mathrm{R}}}\left|u(T)+\chi_{\mathrm{R}} v(T)\right|^{2}+\frac{1}{2} \mathbb{D}_{0} e(u(T)): e(u(T)) \mathrm{d} x+\int_{\Sigma} g \cdot w \mathrm{~d} S \mathrm{~d} t \\
=\int_{Q} \chi_{\mathrm{R}} \mathbb{D}_{0} e(v): e(v) \mathrm{d} x \mathrm{~d} t,
\end{aligned}
$$

where $\mathscr{O}(\tau)$ if from (32). Here we used also that $\sqrt{\varrho} v_{\tau}(T) \rightarrow \sqrt{\varrho} v(T)$ in $L^{2}\left(\Omega ; \mathbb{R}^{d}\right)$, which follows from the second convergence in (27a) together with the estimate (25d). In (34), we used also

$$
\begin{aligned}
& \left|\int_{Q}\left(\mathbb{C}\left(\underline{\alpha}_{\tau}\right)-\mathbb{C}\left(\bar{\alpha}_{\tau}\right)\right) e\left(\dot{u}_{\tau}\right): e\left(\bar{w}_{\tau}\right) \mathrm{d} x \mathrm{~d} t\right| \\
& \quad \leq\left\|\mathbb{C}\left(\underline{\alpha}_{\tau}\right)-\mathbb{C}\left(\bar{\alpha}_{\tau}\right)\right\|_{L^{\infty}\left(Q ; \mathbb{R}^{d^{4}}\right)}\left\|e\left(\dot{u}_{\tau}\right)\right\|_{L^{2}\left(Q ; \mathbb{R}^{d \times d}\right)}\left\|e\left(\bar{w}_{\tau}\right)\right\|_{L^{2}\left(Q ; \mathbb{R}^{d \times d}\right)} \rightarrow 0 .
\end{aligned}
$$

Here we used the first estimates in $(25 \mathrm{a}, \mathrm{b})$ together with the convergence (27c) and the compact embedding of $L^{\infty}\left(I ; W^{1, p}(\Omega)\right) \cap H^{1}\left(I ; L^{2}(\Omega)\right)$ into $C(\bar{Q})$ for $p>d$. This is actually the 
only spot where $p>d$ is vitally needed. The last equality in (34) is the energy conservation in the mechanical-equilibrium part (31).

As we already know $e\left(\underline{\bar{v}}_{\tau}\right) \rightarrow e(v)$ in $L^{2}\left(Q ; \mathbb{R}^{d \times d}\right)$, from (34) we can see even the strong convergence (27d) . Since $e\left(\dot{u}_{\tau}\right)=e\left(\underline{\underline{v}}_{\tau}\right)$, it also says $e\left(\dot{u}_{\tau}\right) \rightarrow e(\dot{u})$, from which the desired strong convergence $e\left(\bar{u}_{\tau}\right) \rightarrow e(u)$ needed for the limit passage in the damage flow rule follows.

Now we can perform the limit passage in the discrete damage flow rule. This is, however, a bit technical. Actually, (21b) means:

$$
\begin{aligned}
\int_{Q} \zeta(z)+\frac{1}{2} \mathbb{C}^{\prime}\left(\bar{\alpha}_{\tau}\right) e\left(\bar{u}_{\tau}\right): e\left(\bar{u}_{\tau}\right)\left(z-\dot{\alpha}_{\tau}\right)-\phi^{\prime}\left(\bar{\alpha}_{\tau}\right)\left(z-\dot{\alpha}_{\tau}\right) \\
+\kappa\left|\nabla \bar{\alpha}_{\tau}\right|^{p-2} \nabla \bar{\alpha}_{\tau} \cdot \nabla\left(z-\dot{\alpha}_{\tau}\right) \mathrm{d} x \mathrm{~d} t \geq \int_{Q} \zeta\left(\dot{\alpha}_{\tau}\right) \mathrm{d} x \mathrm{~d} t
\end{aligned}
$$

Note that, on the time-discrete level, this inequality is indeed legitimate since $\dot{\alpha}_{\tau} \in L^{\infty}(Q)$ so that it is in duality with $\frac{1}{2} \mathbb{C}^{\prime}\left(\bar{\alpha}_{\tau}\right) e\left(\bar{u}_{\tau}\right): e\left(\bar{u}_{\tau}\right) \in L^{1}(Q)$; here we again use $p>d$ although some regularization could relax this requirement at this point. Anyhow, $\dot{\alpha}_{\tau}$ is not uniformly bounded in $L^{\infty}(Q)$ and we must integrate this term by using also the discrete momentum equilibrium to lead (36) closer to the limit inequality (26b). More specifically, we need to use a discrete analog of the identity $\frac{1}{2} \dot{\alpha} \mathbb{C}^{\prime}(\alpha) e(u): e(u)=\frac{\partial}{\partial t} \frac{1}{2} \mathbb{C}(\alpha) e(u): e(u)-\mathbb{C}(\alpha) e(u): e(\dot{u})$ integrated over time as used already in casting (26b) in Definition 4.3. Standardly, this gives the desired inequality if the $(e, \alpha) \mapsto \mathbb{C}(\alpha) e$ : $e$ were convex. In our case, we need to rely only on its (strengthened) semiconvexity: actually, from (24), we can also read the estimate

$$
\frac{\mathscr{E}\left(u_{\tau}^{k}, \alpha_{\tau}^{k}\right)-\mathscr{E}\left(u_{\tau}^{k-1}, \alpha_{\tau}^{k-1}\right)}{\tau} \leq\left\langle\mathscr{E}^{\prime}\left(u_{\tau}^{k}, \alpha_{\tau}^{k}\right), \frac{\left(u_{\tau}^{k}, \alpha_{\tau}^{k}\right)-\left(u_{\tau}^{k-1}, \alpha_{\tau}^{k-1}\right)}{\tau}\right\rangle+\sqrt{\frac{\tau}{\tau_{0}}} \Xi_{0}\left(\frac{u_{\tau}^{k}-u_{\tau}^{k-1}}{\tau}\right) .
$$

When summing it up for $k=1, \ldots, T / \tau$ and writing in terms of the interpolants, we have

$$
\begin{aligned}
\int_{\Omega} \frac{1}{2} \mathbb{C}\left(\alpha_{\tau}(T)\right) e\left(u_{\tau}(T)\right): e\left(u_{\tau}(T)\right)-\frac{1}{2} \mathbb{C}\left(\alpha_{0}\right) e\left(u_{0}\right): e\left(u_{0}\right) \mathrm{d} x \\
\quad \leq \int_{Q} \frac{1}{2} \dot{\alpha}_{\tau} \mathbb{C}^{\prime}\left(\bar{\alpha}_{\tau}\right) e\left(\bar{u}_{\tau}\right): e\left(\bar{u}_{\tau}\right)+\mathbb{C}\left(\bar{\alpha}_{\tau}\right) e\left(\bar{u}_{\tau}\right): e\left(\dot{u}_{\tau}\right)+\sqrt{\frac{\tau}{\tau_{0}}} \mathbb{D}_{0} e\left(\dot{u}_{\tau}\right): e\left(\dot{u}_{\tau}\right) \mathrm{d} x \mathrm{~d} t
\end{aligned}
$$

Due to the estimate of $e\left(\dot{u}_{\tau}\right)$ in $L^{2}\left(Q ; \mathbb{R}^{d \times d}\right)$, the last term (37) converges to 0 as $\mathcal{O}(\sqrt{\tau})$.

A similar note is about the term $\kappa\left|\nabla \bar{\alpha}_{\tau}\right|^{p-2} \nabla \bar{\alpha}_{\tau} \cdot \nabla \dot{\alpha}_{\tau}$ whose treatment is however simpler, namely $\int_{\Omega} \frac{\kappa}{p}\left|\nabla \alpha_{\tau}(T)\right|^{p}-\frac{\kappa}{p}\left|\nabla \alpha_{0}\right|^{p} \mathrm{~d} x \leq \int_{Q} \kappa\left|\nabla \bar{\alpha}_{\tau}\right|^{p-2} \nabla \bar{\alpha}_{\tau} \cdot \nabla \dot{\alpha}_{\tau} \mathrm{d} x \mathrm{~d} t$. By all these estimates, (36) yields also

$$
\begin{aligned}
& \int_{Q} \zeta(z)+\left(\frac{1}{2} \mathbb{C}^{\prime}\left(\bar{\alpha}_{\tau}\right) e\left(\bar{u}_{\tau}\right): e\left(\bar{u}_{\tau}\right)-\phi^{\prime}\left(\bar{\alpha}_{\tau}\right)\right) z+\kappa\left|\nabla \bar{\alpha}_{\tau}\right|^{p-2} \nabla \bar{\alpha}_{\tau} \cdot \nabla z \mathrm{~d} x \mathrm{~d} t \\
& \geq \int_{Q} \zeta\left(\dot{\alpha}_{\tau}\right)+\mathbb{C}\left(\bar{\alpha}_{\tau}\right) e\left(\bar{u}_{\tau}\right): e\left(\dot{u}_{\tau}\right) \mathrm{d} x \mathrm{~d} t+\int_{\Omega}\left(\frac{1}{2} \mathbb{C}\left(\alpha_{\tau}(T)\right) e\left(u_{\tau}(T)\right): e\left(u_{\tau}(T)\right)\right. \\
&\left.\quad-\phi\left(\alpha_{\tau}(T)\right)+\frac{\kappa}{p}\left|\nabla \alpha_{\tau}(T)\right|^{p}-\frac{1}{2} \mathbb{C}\left(\alpha_{0}\right) e\left(u_{0}\right): e\left(u_{0}\right)+\phi\left(\alpha_{0}\right)-\frac{\kappa}{p}\left|\nabla \alpha_{0}\right|^{p}\right) \mathrm{d} x
\end{aligned}
$$

The limit passage towards the inequality $(26 \mathrm{~b})$ is then easy by weak (lower) semicontinuity, using also that $e\left(u_{\tau}(T)\right) \rightarrow e(u(T))$ in $L^{p}\left(\Omega ; \mathbb{R}^{d \times d}\right)$ and $\nabla \alpha_{\tau}(T) \rightarrow \nabla \alpha(T)$ in $L^{p}\left(\Omega ; \mathbb{R}^{d}\right)$. 
Remark 4.5 (Convergence of iterative procedures.) When applying a (here unspecified) conformal finite-element space discretisation for the boundary-value problem (10)(11), it becomes equivalent to a minimization of (17) on the respective finite-dimensional subspace $H^{1}\left(\Omega ; \mathbb{R}^{d}\right) \times H^{1}(\Omega)$ respecting also the (discretised) constraints $0 \leq \alpha \leq \alpha_{\tau}^{k-1}$. This leads to a convex mathematical-programming problem. The iterative procedures of the Newton-Raphson type (which are in the potential case then identical with sequential quadratic programming) enjoys a global guaranteed convergence to the only one global minimizer of (17). More in detail, these methods need a globalization (so-called trust-region strategy) in general, but when the positive-definite Hessian has a bounded condition number, a global convergence is ensured provided the step-length used in particular iterations is controlled appropriately, cf. e.g. the monographs [12, 36,50]. For this, we need here that the time-step criterion (19) is satisfied. Let us note that (17) is then uniformly convex and the Hessian can effectively be considered bounded. For this last attribute, one should consider the constraints on $\alpha$ valued in the interval $[0,1]$ and, because of the term $\gamma(\alpha) \mathbb{C}_{1} e(u): e(u)$, also the constraints on $e(u)$ which is a-priori bounded in $L^{2}\left(\Omega ; \mathbb{R}^{d \times d}\right)$ uniformly due to the estimate (25c) and, when considering a fixed space discretisation, also in $L^{\infty}\left(\Omega ; \mathbb{R}^{d \times d}\right)$. Thus the condition number of the Hessian of (17) is effectively bounded and iterative methods enjoy (at least theoretically) global convergence on the mentioned trust region, although it may be very ill-conditioned for fine space discretisations.

Remark 4.6 (Uniqueness issue.) The uniquenees of the weak solution to the system (9), which would in particular guarantee convergence of the whole sequence (not only selected subsequences) in Proposition [4.4), seems open. Some results can be found e.g. in [24, Prop. 7.5.5] but it requires $\mathbb{D}$ independent of $\alpha$. On the other hand, a possible nonuniqueness is even a desired phenomenon reflecting high sensitivity and unstability in real experiments typically occuring in damage and fracture mechanics.

\section{Concluding remarks}

Some special cases of the model and various modifications the model are worth mentioning:

Remark 5.1 (Phase-field fracture.) The concept of bulk damage can (approximately) imitate the philosophy of fracture along surfaces if the damage stored energy $\phi^{\prime}$ is big. The popular ansatz is

$$
\varphi_{\mathrm{E}}(e, \alpha, \nabla \alpha):=\gamma(\alpha) \mathbb{C} e: e+\underbrace{G_{\mathrm{c}}\left(\frac{1}{2 \varepsilon}(1-\alpha)^{2}+\frac{\varepsilon}{p}|\nabla \alpha|^{p}\right)}_{\text {crack surface density }} \text { with } \gamma(\alpha)=\frac{\left(\varepsilon / \varepsilon_{0}\right)^{2}+\alpha^{2}}{2}
$$

with $\varepsilon_{0}>0$ and $G_{\mathrm{c}}>0$ fixed. For $p=2$, this is known as the so-called Ambrosio-Tortorelli functional. In the static case, this approximation was proposed in [1,2] for the scalar case and the asymptotic analysis for $\varepsilon \rightarrow 0$ was rigorously executed. The generalization for the vectorial case is due to Focardi [15]. Later, it was extended for evolution situation, namely for a rate-independent cohesive damage in [18], see also also [8, 2, 25, 32] where also inertial 
forces are sometimes considered. It is vastly used in engineering under the name a phase-field model, cf. e.g. [31, 45, 51, 53] where also various modifications of the stored energy (39) and various dissipation potentials have been devised, although without any rigorous analytical justification of the resulted models. In fact, this fits with (1a) with $\phi(\alpha)=-G_{\mathrm{c}}(1-\alpha)^{2} /(2 \varepsilon)$ and $\kappa=\varepsilon G_{\mathrm{c}}$. The dissipation $\zeta=\zeta(\dot{\alpha})$ is considered zero for $\dot{\alpha} \leq 0$ and $+\infty$ otherwise. When accompanied by viscosity, it fits with the assumptions (6a) and (13a). For a small length-scale parameter $\varepsilon>0$, this model allows for a nearly complete damage and with a tendency to be localized on very small volumes along evolving surfaces where the cracks propagates, while elsewhere $\alpha \sim 1$ not to make the term $(1-\alpha)^{2} / \varepsilon$ too large. By this way, it approximates the infinitesimally thin cracks and in particular their propagation. However, it is well known that such phase-field model (as well as Griffith fracture model) does not work well for initiation of cracks unless some notches are presented, cf. e.g. the discussion in [51]. Some attempt for improvement consists in nonquadratic degradation function $\gamma$, cf. [11, 45, 53]. The monolithic discretisation is particularly suitable for such modification because it does not rely on componentwise quadratic structure of (39) for $p=2$, in contrast to staggered schemes with linear-quadratic solvers. On the other hand, to achieve the mentioned good initiation and propagation and simultaneously allow for small values of $\varepsilon$, one should choose $\gamma$ such that $\gamma^{\prime}(1)=\mathcal{O}(1 / \varepsilon)$ but then $\tau_{0}$ in the criterion (19) is as $\mathcal{O}\left(\varepsilon^{2}\right)$ for $\varepsilon \rightarrow 0$, which makes this restriction quite strong.

Remark 5.2 (General $\phi^{\prime}$ 's.) For a non-concave $\phi$, one can modify (10c) by replacing $\phi^{\prime}\left(\alpha_{\tau}^{k}\right)$ by the difference quotient $\left(\phi\left(\alpha_{\tau}^{k}\right)-\phi\left(\alpha_{\tau}^{k-1}\right)\right) /\left(\alpha_{\tau}^{k}-\alpha_{\tau}^{k-1}\right)$ where-ever $\alpha_{\tau}^{k} \neq \alpha_{\tau}^{k-1}$. The approximate energetics (22) as well as the a-priori estimates (25) keep holding, as well as the existence of a potential behind the incremental problems, cf. [43].

Remark 5.3 (Purely elastic models.) The inertial term itself helps to convexify the incremental problems but, in contrast to the Kelvin-Voigt viscosity, this stabilization is dependent also on the spatial discretisation. Typically, the ratio between conditional numbers of the mass and the elasticity operator $\operatorname{div}(\mathbb{C} e(\cdot))$ after space discretisation is $\mathscr{O}\left(h^{2}\right)$ with $h>0$ the mesh size of the spatial discretisation. This makes $\tau_{0}$ in the criterion (19) with $\mathbb{D}_{0}$ replaced by the mass matrix dependent on $h$ as $\mathscr{O}\left(h^{2}\right)$ for $h \rightarrow 0$, which would force for practically unbearably too small time steps. On top of it, the convergence analysis of purely elastic model requires to enhance the model by strain gradients, cf. [24, Sect. 7.5.3], which is sometimes used in the context of damage mechanics to model dispersion of elastic waves [5]. Moreover, sometimes even inertia gradients are used, see again [5], which then would have the same mesh-independent convexifying effect as the Kelvin-Voigt viscosity.

Remark 5.4 (Plasticity, creep, phase transformations, mass transfer.) Let us emphasize that one can relatively routinely combine the above presented damage (or phasefield fracture) model with other phenomena when involving some other internal variables as plastic/creep strain, or volume fraction of various phases (e.g. in shape-memory materials), or concentration in poro-elastic materials. Then one immediately gets monolithic schemes for a combination with plasticity (i.e. so-called ductile damage or fracture, in contrast to brittle) or visco-elastic creep rheology, or for a combination with a diffusant flow in damageable poro-visco-elastic media. 


\section{Acknowledgment}

The author is deeply thankful for the hospitality of the University of Seville during his stays in 2017-8 and for inspiring discussions with José Reinoso. The discussion with Ladislav Lukšan about optimization algorithms and the comments of an anonymous referee are highly appreciated. Also the partial support from the Czech Science Foundation through the grant 16-34894L "Variational structures in continuum thermomechanics of solids", 17-04301S "Advanced mathematical methods for dissipative evolutionary systems", 18-03834S "Localization phenomena in shape memory alloys", and 19-04956S "Dynamic and nonlinear behaviour of smart structures; modelling and optimization", and also the institutional support RVO: 61388998 (ČR) are acknowledged.

\section{References}

[1] L. Ambrosio and V. M. Tortorelli. On the approximation of free discontinuity problems. Bollettino Unione Mat. Italiana, 7:105-123, 1992.

[2] L. Ambrosio and V.M. Tortorelli. Approximation of functional depending on jumps via by elliptic functionals via $\Gamma$-convergence. Comm. Pure Appl. Math., 43:999-1036, 1990.

[3] Z.P. Bažant and M. Jirásek. Nonlocal integral formulations of plasticity and damage: Survey of progress. J. Engr. Mech. ASCE, 128:1119-1149, 2002.

[4] A. Bedford. Hamilton's Principle in Continuum Mechanics. Pitman, Boston, 1985.

[5] T. Bennett, A. Rodríguez-Ferran, and H. Askes. Damage regularisation with inertia gradients. Europ. J. Mech. - A/Solids, 31:131-138, 2012.

[6] M.J. Borden, C.V. Verhoosel, M.A. Scott, T.J.R. Hughes, and C.M. Landis. A phase-field description of dynamic brittle fracture. Comp. Meth. Appl. Mech. Engr., 217-220:77-95, 2012.

[7] B. Bourdin. Numerical implementation of the variational formulation for quasi-static brittle fracture. Interfaces Free Bound., 9:411-430, 2007.

[8] B. Bourdin, G.A. Francfort, and J.-J. Marigo. The variational approach to fracture. J. Elasticity, 91:5-148, 2008.

[9] B. Bourdin, C.J. Larsen, and C.L. Richardson. A time-discrete model for dynamic fracture based on crack regularization. Int. J. of Fracture, pages 133-143, 2011.

[10] S. Burke, C. Ortner, and E. Süli. An adaptive finite element approximation of a variational model of brittle fracture. SIAM J. Numer. Anal., 48:980-1012, 2010.

[11] S. Burke, C. Ortner, and E. Süli. An adaptive finite element approximation of a generalised Ambrosio-Tortorelli functional. Math. Models Methods Appl. Sci., 23:1663-1697, 2013. 
[12] A.R. Conn, N.I.M. Gould, and P.L.Toint. Trust-Region Methods. SIAM/MPS, Philadelphia, 2000.

[13] R. Graca e Costa, J. Alfaiate, D. Dias da Costa, and L.J. Sluys. A non-iterative approach for the modelling of quasi-brittle materials. Intl. J. Fracture, 178:281-298, 2012.

[14] P. Farrell and C. Maurini. Linear and nonlinear solvers for variational phase-field models of brittle fracture. Int. J. Numer. Meth. Engng., 109:648-667, 2017.

[15] M. Focardi. On the variational approximation of free-discontinuity problems in the vectorial case. Math. Models Methods Appl. Sci., 11:663-684, 2001.

[16] M. Frémond. Non-Smooth Thermomechanics. Springer-Verlag, Berlin, 2002.

[17] L.B. Freund. Dynamic Fracture Mechanics. Cambridge Univ. Press, Cambridge, 1998.

[18] A. Giacomini. Ambrosio-Tortorelli approximation of quasi-static evolution of brittle fractures. Calc. Var. Partial Diff. Eqs., 22:129-172, 2005.

[19] Y. Hamiel, V. Lyakhovsky, and Y. Ben-Zion. The elastic strain energy of damaged solids with applications to non-linear deformation of crystalline rocks. Pure Appl. Geophys., 2011.

[20] L.M. Kachanov. Time of rupture process under Deep conditions. Izv. Akad. Nauk SSSR, 8:26, 1958.

[21] L.M. Kachanov. Introduction to Continuum Damage Mechanics. Kluwer, 2nd edition, 1990.

[22] D. Knees and M. Negri. Convergence of alternate minimization schemes for phase field fracture and damage. Math. Models Meth. Appl. Sci., 27:1743-1794, 2017.

[23] N. Knight Jr., C. Rankin, and F. Brogan. Controlling progressive failure analyses using artificial viscous damping. In 19th AIAA Applied Aerodynamics Conference Anaheim, $C A$. Amer. Inst. Aeronautics \& Astronautics, 2001.

[24] M. Kružík and T. Roubíček. Mathematical Methods in Continuum Mechanics of Solids. Springer, Switzerland, 2019, in print, ISBN 978-3-030-02064-4.

[25] C.J. Larsen, C. Ortner, and E. Süli. Existence of solution to a regularized model of dynamic fracture. Math. Models Meth. Appl. Sci., 20:1021-1048, 2010.

[26] G. Liu, Q. Li, M.A. Msekh, and Z. Zuo. Abaqus implementation of monolithic and staggered schemes for quasi-static and dynamic fracture phase-field model. Computational Materials Science, 121:35-47, 2016.

[27] V. Lyakhovsky, Y. Hamiel, and Y. Ben-Zion. A non-local visco-elastic damage model and dynamic fracturing. J. Mech. Phys. Solids, 59:1752-1776, 2011.

[28] P. Maimí, P.P. Camanho, J.A. Mayugo, and C.G. Dávila. A continuum damage model for composite laminates: part I/II. Mech. Mater., 39:897-908,909-919, 2007. 
[29] G.A. Maugin. The Thermomechanics of Plasticity and Fracture. Cambridge University Press, Cambridge, 1992.

[30] C. Miehe, F. Welschinger, and M. Hofacker. A phase field model for rate-independent crack propagation: Robust algorithmic implementation based on operator splits. Computer Meth. Appl. Mech. Engr., 199:2765-2778, 2010.

[31] C. Miehe, F. Welschinger, and M. Hofacker. Thermodynamically consistent phase-field models of fracture: Variational principles and multi-field FE implementations. Intl. J. Numer. Meth. Engr., 83:1273-1311, 2010.

[32] A. Mielke and T. Roubíček. Rate Independent Systems - Theory and Application. Springer, New York, 2015.

[33] G. Molnár and A. Gravouil. 2D and 3D Abaqus implementation of a robust staggered phase-field solution for modeling brittle fracture. Finite Elements in Analysis and Design, 130:27-38, 2017.

[34] M.A. Msekh, J.M. Sargado, M. Jamshidian, P.M. Areias, and T. Rabczuk. Abaqus implementation of phase-field model for brittle fracture. Comput. Mater. Sci., 96 B:472484, 2015.

[35] P. Neto, J. Alfaiate, and T. Domingues. Numerical modelling of concrete beams reinforced with pre-stressed CFRP. Intl. J. Fract., 157:159-173, 2009.

[36] J. Nocedal and S.J. Wright. Numerical Optimization. Springer, New York, 2nd edition, 2006.

[37] M. Paggi, M. Corrado, and J. Reinoso. Fracture of solar-grade anisotropic polycrystalline Silicon: A combined phase field-cohesive zone model approach. Comp. Meth. Appl. Mech. Engr., 330:123-148, 2018.

[38] M. Paggi and J. Reinoso. Revisiting the problem of a crack impinging on an interface: a modeling framework for the interaction between the phase field approach for brittle fracture and the interface cohesive zone model. Comp. Meth. Appl. Mech. Engr., 321:145-172, 2017.

[39] T. Roubíček. Rate independent processes in viscous solids at small strains. Math. Methods Appl. Sci., 32:825-862 (Erratum p. 2176), 2009.

[40] T. Roubíček. Nonlinear Partial Differential Equations with Applications. Birkhäuser, Basel, 2nd edition, 2013.

[41] T. Roubíček. An energy-conserving time-discretisation scheme for poroelastic media with phase-field fracture emitting waves and heat. Disc. Cont. Dynam. Syst. S, 10:867893, 2017.

[42] T. Roubíček. Models of dynamic damage and phase-field fracture, and their various time discretisations. In J.-F. Rodrigues and M. Hinttermüller, editors, Topics in Applied Analysis and Optimisation, CIM Series in Math. Sci. Springer, to appear. 
[43] T. Roubíček and C.G. Panagiotopoulos. Energy-conserving time-discretisation of abstract dynamical problems with applications in continuum mechanics of solids. Numer. Funct. Anal. Optim., 38:1143-1172, 2017.

[44] Y.A. Roy and R.H. Dodds Jr. Simulation of ductile crack growth in thin aluminum panels using 3-D surface cohesive elements. Intl. J. Fracture, 110:21-45, 2001.

[45] J.M. Sargado, E. Keilegavlen, I. Berre, and J.M. Nordbotten. High-accuracy phase-field models for brittle fracture based on a new family of degradation functions. J. Mech. Phys. Solids, 111:458-489, 2018.

[46] M. Shillor, M. Sofonea, and J.J. Telega. Models and Analysis of Quasistatic Contact. Springer, Berlin, 2004.

[47] M. Sofonea, W. Han, and M. Shillor. Analysis and approximation of contact problems with adhesion or damage. Chapman \& Hall/CRC, Boca Raton, FL, 2006.

[48] S.H. Song, G.H. Paulino, and W.G. Buttlar. A bilinear cohesive zone model tailored for fracture of asphalt concrete considering viscoelastic bulk material. Engr. Fracture Mech., 73:2829-2848, 2006.

[49] S.H. Song, G.H. Paulino, and W.G. Buttlar. Simulation of crack propagation in asphalt concrete using an intrinsic cohesive zone model. J. Engr. Mech., 132:1215-1223, 2006.

[50] W. Sun and Y.-X. Yuan. Optimization Theory and Methods: Nonlinear Programming. Springer, New York, 2006.

[51] E. Tanné, T. Li, B. Bourdin, J.-J. Marigo, and C. Maurini. Crack nucleation in variational phase-field models of brittle fracture. J. Mech. Phys. Solids, 110:80-99, 2018.

[52] R.L. Williamson and D.A. Knoll. Simulating dynamic fracture in oxide fuel pellets using cohesive zone models. In 20th Intl. Conf. Structural Mechanics in Reactor Technology, page Paper 1775, Espoo, Finland, 2009.

[53] J.-Y. Wu and V.P. Nguyen. A length scale insensitive phase-field damage model for brittle fracture. J. Mech. Phys. Solids, 119:20-42, 2018. 